\title{
Fiber optics for spin waves
}

\author{
Xiangjun Xing ${ }^{1}$ and Yan Zhou ${ }^{2}$ \\ Magnetic skyrmions in chiral magnets with the Dzyaloshinskii-Moriya interaction have received intensive attention because of \\ their potential in prospective applications. Here, we theoretically demonstrate that another novel spin texture in chiral magnets- \\ the chiral strip-domain wall (SDW) — can generate a deep one-dimensional potential well of magnetic origin. We show by \\ micromagnetic simulations that the potential well caused by a SDW can serve as an internal channel to guide spin-wave (SW) \\ propagation, which makes the ultrathin chiral magnet including the SDW become a reconfigurable self-cladding optic-fiber-like \\ magnonic waveguide with a graded refractive index. Furthermore, we design logical NOT and NAND gates based on the state- \\ modulated transmission property of the magnonic waveguide. We also reveal that a SDW can be reliably written into the gate \\ arms using the Slonczewski spin torque. Finally, prospective applications of the observed potential well in other fields are \\ envisioned. This work is expected to open new possibilities for SW guiding and manipulation in ultrathin magnetic \\ nanostructures as well as to help shape the field of beam magnonics.
}

NPG Asia Materials (2016) 8, e246; doi:10.1038/am.2016.25; published online 18 March 2016

\section{INTRODUCTION}

Magnonic circuits are estimated to be capable of providing substantial throughput enhancement compared with those circuits currently based on complementary metal-oxide-semiconductor technology; special tasks such as advanced image processing and speech recognition that benefit from parallel processing of information will also be possible. $^{1,2}$ To achieve multifunctionality of magnonic circuits, controlled propagation and manipulation of spin waves (SWs) in a rich variety of magnetic nanostructures are required.

With the continued downscaling of magnetic films, the antisymmetric Dzyaloshinskii-Moriya interaction (DMI) ${ }^{3,4}$ arising from spinorbit scattering of itinerant electrons has manifested its roles in ultrathin samples with inversion-asymmetric interfaces, ${ }^{5,6}$ where various spin textures such as spin spirals, ${ }^{7}$ chiral domain walls ${ }^{8,9}$ and skyrmions ${ }^{10}$ were observed recently. As already shown in (refs 11-15), inhomogeneous spin textures may offer efficient means for tailoring spin-wave (SW) propagation in magnetic nanostructures. Consequently, ultrathin nanostructures with interface-induced DMI will become promising candidates for the construction of magnonic devices with versatile functionalities. ${ }^{1,16-18}$ So far, guided propagation and manipulation of SWs in chiral nanostructures containing unique spin textures have not yet been addressed, despite their strong relevance to potential applications of chiral magnets in magnonics as well as to understanding the strength of relevant interactions. ${ }^{19,20}$ In the widely used Damon-Eshbach (DE) propagation geometry, ${ }^{21}$ SWs in a strip-type waveguide-regardless of the center or edge modes ${ }^{22}$ have nonzero precession amplitude at the boundary; ${ }^{23}$ therefore, they might suffer from undesired scattering caused by boundary irregularities that have been found to result in reduced attenuation length. ${ }^{22}$ The self-cladding waveguide with well-defined internal channels suggested by Duerr et al. ${ }^{13}$ can resolve the edge-scattering problem, but the channels must be maintained by an applied field, which is not preferred in real devices.

In this work, we demonstrate the realization of a novel type of graded-refractive-index magnonic waveguides with self-cladding internal nanochannels, in which SWs are trapped transversely and propagate an ultra-narrow beam less than $10 \mathrm{~nm}$ in beamwidth. The channeling effect stems from the strong inhomogeneity of the internal field in the direction normal to the strip-domain wall (SDW) stretching direction. The drastic decrease in this field inside the SDWs creates a narrow potential well that is deep enough to allow precession of magnetization at ultralow frequencies. In addition, multichannel SW propagation in a single waveguide is accomplished by introducing multiple SDWs into the waveguide (such a multi-SDW spin texture should exist ubiquitously in magnetic films with interfacial DMI, as has been observed in a few samples made of different materials and/or structures). ${ }^{9,24,25}$ Furthermore, we show that when the waveguide is toggled to a quasiuniform single-domain state, no SWs can enter and pass through the waveguide unless the frequency is increased to tens of gigahertzes. Finally, we implement logic-NOT and -NAND gates by virtue of the observed state-modulated SW transmission, and we demonstrate how a SDW can be reliably written into the gate's arm to ensure the feasibility of this logic architecture. The advantage lies in its complete compatibility with racetrack memory, ${ }^{26}$ that is, it can be

\footnotetext{
${ }^{1}$ Institute of Micro-Nano Structures and Optoelectronics, College of Physics and Electronic Information Engineering, Wenzhou University, Wenzhou, China and ${ }^{2}$ School of Electronic Science and Engineering and Collaborative Innovation Center of Advanced Microstructures, Nanjing University, Nanjing, China

Correspondence: Professor Y Zhou, School of Electronic Science and Engineering and Collaborative Innovation Center of Advanced Microstructures, Nanjing University, Nanjing 210093, China.

E-mail: yanzhouy@hotmail.com

Received 6 July 2015; revised 20 November 2015; accepted 17 December 2015
} 
directly interconnected with the latter circuits because the unit (magnonic waveguide) used in these gates can, after proper reconfiguration, work as a nanotrack for skyrmion motion in skyrmion-based racetrack memories. ${ }^{27,28}$

\section{MATERIALS AND METHODS}

\section{Micromagnetic models}

The layout of the present study is shown in Figure la. The magnonic waveguide, in which SWs will be excited and guided, is composed of a rectangular magnetic plate elongated spatially in $x$ direction. It should be patterned from an ultrathin magnetic film with inversion-symmetry-breaking interfaces to ensure the occurrence of an induced $\mathrm{DMI}^{5}$ required to stabilize chiral SDWs in the waveguide. In the following sections, we will demonstrate, as a first step, the fundamental principle of utilizing a SDW to channel SW propagation in a magnonic waveguide. Here, we chose a 1200-nm-long and 60-nm-wide waveguide, in which a single SDW is included (Figures 1,2,3 and Supplementary Figures 1). For comparison, however, we also checked SW propagation in the same waveguide with a distinct spin configuration. Subsequently, we will show that SW propagation along separate channels in parallel is attainable in a single waveguide. For this, the additional widths of 120 and $160 \mathrm{~nm}$ were adopted to accommodate more than one SDW (Figure 4). Finally, we will illustrate how to perform logic operations using the statemodulated SW output. To this end, a waveguide of $300 \mathrm{~nm}$ long and $60 \mathrm{~nm}$ wide was employed as the basic unit of the logic gates (Figures 5 and 6). All waveguides are $1 \mathrm{~nm}$ thick.

\section{Numerical simulations}

Micromagnetic simulations ${ }^{29}$ with MuMax $^{30}$ and OOMMF $^{31}$ codes were carried out to seek a static equilibrium spin configuration in the magnonic a

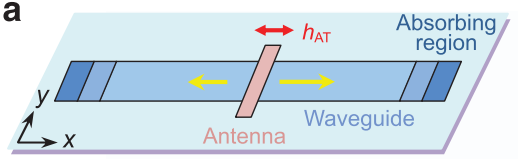

C

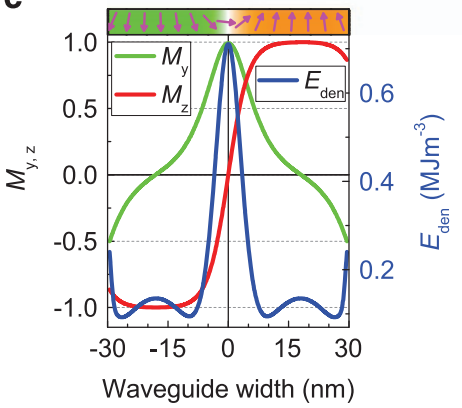

b
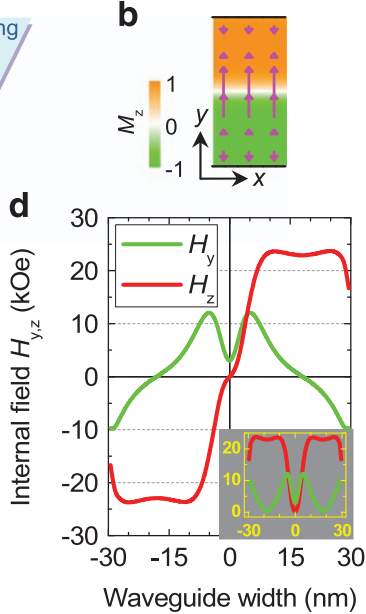

Figure 1 Setup for SW excitation and propagation, SDW state and its internal-field profile. (a) The waveguide is made of chiral magnetic films with an interfacial DMI. A microstrip antenna is fixed at the center of and perpendicularly to the waveguide, so that the spin waves, once emitted after opening the excitation field $h_{\mathrm{AT}}$, will travel in opposite directions as indicated by the yellow arrows. The areas in dark blue at the ends of the waveguide form an absorbing boundary used to suppress SW reflection. The waveguide is $1200 \mathrm{~nm}$ in length and can have different widths depending on the number of SDWs that are intended for writing. (b) Spin configuration of the SDW state in a 60-nm-wide waveguide. The SDW sits between two strip domains with up and down magnetization. The SDW has a definite chirality (anticlockwise herein) as determined by the DMI. (c) Cross-section view of the above spin configuration and the magnetization and energydensity distribution over the waveguide width. (d) The internal-field distribution over the waveguide width. The magnitude of these fields shown in the inset indicates that a deep potential well is formed in the center of the waveguide. The well width, $\sim 10 \mathrm{~nm}$, is defined as the full-width at halfmaximum (FWHM) of the well in $\mathrm{H}_{\mathrm{z}}$. waveguide and to trace the dynamics of magnetization driven by an external stimulus. For all computations, the Landau-Lifshitz-Gilbert equation was augmented with the interfacial DMI term, ${ }^{32}$ and for those simulations examining the spin dynamics triggered by an out-of-plane electric current (Figure 6), the Slonczewski spin torque ${ }^{33}$ (together with the DMI) was added into the Landau-Lifshitz-Gilbert equation as well. For finite-temperature simulations, the random thermal field of the Brown form ${ }^{30,34}$ was included in the effective field (the results are shown in Supplementary Figure 4). The micromagnetic simulation details are presented in the Supplementary Information. The material parameters adopted in the simulations are typical of $\mathrm{Pt} / \mathrm{Co}$ systems with perpendicular magnetic anisotropy: ${ }^{27}$ the saturation magnetization $M_{\mathrm{s}}=580 \mathrm{kA} \mathrm{m}^{-1}$, the exchange stiffness $A=15 \mathrm{pJ} \mathrm{m}^{-1}$ and the Gilbert damping was assumed to be $\alpha=0.01^{27,35,36}$ throughout the waveguide except at the absorbing boundary region, ${ }^{37}$ where its value is greatly enhanced ( $\alpha=0.05^{38-40}$ was also examined in the simulations, which gave similar results as $\alpha=0.01$; see Supplementary Figure 3). One apparent difference is that the SWs decay expectedly faster for $\alpha=0.05$ than for $\alpha=0.01$. The details about the choice of the damping values are shown in the Supplementary Information). A series of values in large intervals were examined for $K_{\mathrm{u}}$ (the perpendicular magnetocrystalline anisotropy) and $D$ (the DMI strength) to ensure the validity of the results to a variety of possible samples (Supplementary Figures 1 and 2). For many of the combinations of $K_{\mathrm{u}}$ and $D$, the waveguide can stay in a multiSDW state where opposite strip domains are separated by chiral SDWs. The results presented in the figures throughout the paper correspond to $K_{\mathrm{u}}=0.8 \mathrm{MJ} \mathrm{m}^{-3}$ (thereby, the effective uniaxial anisotropy $K_{\text {eff }}=0.6 \mathrm{MJ} \mathrm{m}^{-3}$ as determined from $\left.K_{\text {eff }}=K_{\mathrm{u}^{-}}(1 / 2) \mu_{0} M_{\mathrm{s}}^{2}\right)^{27,41}$ and $D=3.5 \mathrm{MJ} \mathrm{m}^{-2}$. The computational volume is discretized into cubic meshes of $1 \times 1 \times 1 \mathrm{~nm}^{3}$ irrespective of the sample size. The excitation field $h_{\mathrm{AT}}$ is applied to an area of $4 \times w \mathrm{~nm}^{2}(w$ is the waveguide width) in the waveguide specified by the antenna, and it has a time profile of either $\boldsymbol{h}_{\mathrm{AT}}(t)=H_{0} \cdot \sin \left[2 \pi f_{\mathrm{c}}\left(t-t_{0}\right)\right] /\left[2 \pi f_{\mathrm{c}}\left(t-t_{0}\right)\right] \hat{e}_{\mathrm{x}}$, where $H_{0}=2000 \mathrm{Oe}$ and $f_{\mathrm{c}}=80 \mathrm{GHz}$ are the amplitude and cutoff frequency of the sinc field, or $\boldsymbol{h}_{\mathrm{AT}}(t)=h_{0} \cdot \sin (2 \pi f t) \hat{\mathrm{x}}_{\mathrm{x}}$, where $h_{0}$ and $f$ are the amplitude and frequency of the $r f$ field, for different purposes. For $h_{0}=10$ Oe and 200 Oe, no significant difference in the results was observed, except that the 200 Oe field can render higher phase resolution between neighboring simulation cells. In addition, $h_{0}=1000$ Oe and 10000 Oe were used to check the stability of the SDW state; it was found that the SDW structure could not be destroyed at these fields.

Code availability. OOMMF and MuMax3 used to generate the results are open-source, public-domain codes and can be accessed freely at http://math. nist.gov/oommf/ (Release version 1.2a5) and http://mumax.github.io/ (Release version 3), respectively.

\section{RESULTS}

Formation of a deep one-dimensional (1D) potential well along the SDW

In Figures 1b, a SDW is placed between the top and bottom strip domains with up and down magnetization, respectively. The side view of the spin texture is shown in Figure 1c. It is clear that the SDW is of Neel type ${ }^{8}$ and has a left-handed chirality (along the $+y$ axis, the magnetization rotates counterclockwise from downward to upward) guaranteed by the interfacial DMI. Here, the roles of the DMI are twofold: it stabilizes a twisted spin configuration and the chirality of the twisted magnetization. Without the DMI, it is impossible to realize a spin texture in such a small sample. The variation of $M_{\mathrm{y}}$ and $M_{\mathrm{z}}$ over the waveguide width, plotted in Figure 1c, indicates that the profile of $M_{\mathrm{y}}$ is symmetric about the SDW line, whereas the $M_{\mathrm{z}}$ profile is antisymmetric. The SDW state is protected by its own spin configuration with such an alignment of the $M_{\mathrm{y}}$ component. As the middle SDW approaches any of the lateral edges, it feels increased repulsive force from that edge similar to the situation encountered by skyrmions moving toward one edge of a racetrack. ${ }^{27,38}$

The magnetic energy landscape across the waveguide width, corresponding to the shown configuration, is plotted in Figure 1b. 
a

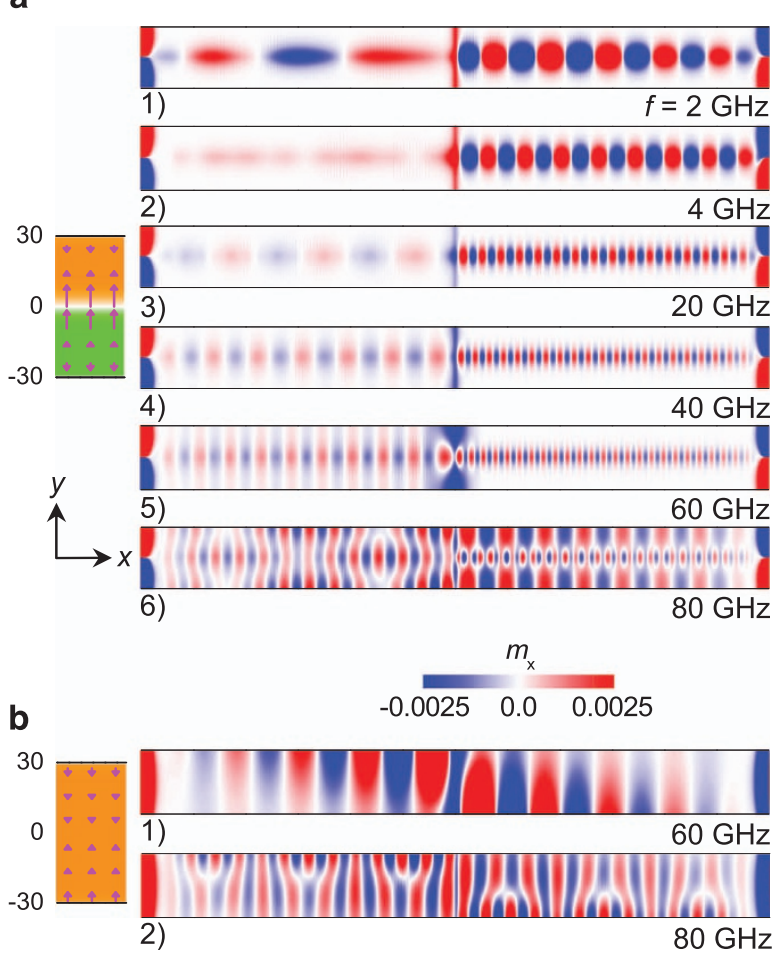

C

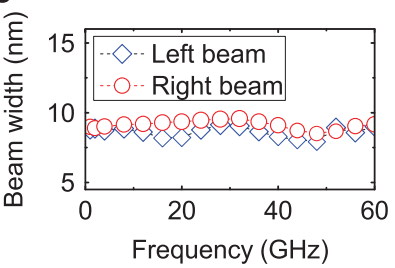

d
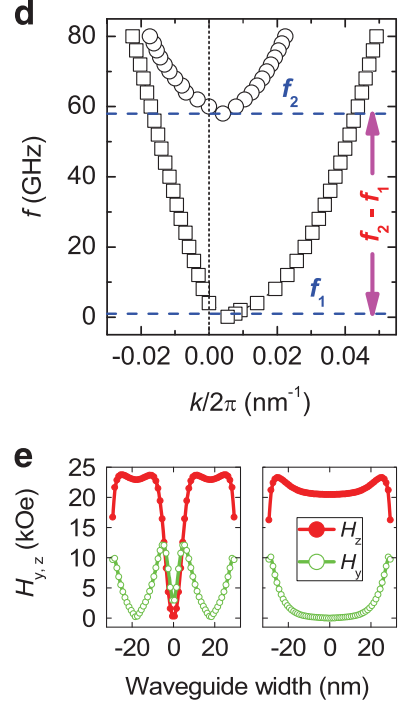

Figure 2 Channeled SW propagation, wave characteristics and confining potentials for the SDW and single-domain states. SW propagation patterns at specified frequencies in the 60-nm-wide waveguide for the (a) SDW and (b) single-domain states. (c) The beamwidth as a function of frequency. The beamwidth is defined as the FWHM of the SW amplitude distribution over the waveguide width. (d) SW dispersion relation. The lower branch corresponds to the well-mode SWs along the center channel of the SDW state, and the upper branch is for the edge-mode SWs along the edge channels ${ }^{14}$ of the singledomain state. $f_{1}$ and $f_{2}$ represent the open frequencies of the well-mode and edge-mode SWs. (e) Internal-field landscape for the SDW and single-domain states. The center well caused by the presence of the SDW is about six times deeper than the edge wells arising from the partial walls ${ }^{14}$ at the lateral edges. This difference in the depth of these wells can explain the distinct $f_{1}$ and $f_{2}$ values.

By recalling that the effective field $\boldsymbol{H}_{\text {eff }}$ is the partial derivative of the energy density $E$ with respect to the magnetization, that is, $\boldsymbol{H}_{\mathrm{eff}} \sim-\partial E /$ $\partial \boldsymbol{m}$, the effective magnetic field associated with the spin texture can be obtained as plotted in Figure 1d. Clearly, the $H_{\mathrm{y}}\left(H_{\mathrm{z}}\right)$ component of the effective field has the same symmetry as the $M_{\mathrm{y}}\left(M_{\mathrm{z}}\right)$ magnetization component. However, the field distribution exhibits enhanced inhomogeneity compared with the magnetization distribution. If we focus mainly on the magnitudes of $H_{\mathrm{y}}$ and $\mathrm{H}_{\mathrm{z}}$ but disregard their signs, we obtain the field profile as inserted in Figure 1d, from which the formation of a potential well can be clearly observed. The potential well created by the field inhomogeneity is centered at $y=0$ (that is, along the SDW line). Apart from the minimum at $y=0$, there are two other minima in $H_{\mathrm{y}}$ at $y \sim \pm 20 \mathrm{~nm}$, where no potential wells can be induced yet because $H_{\mathrm{z}}$ is close to its maximum. A potential well can be formed only at the position where both $H_{\mathrm{y}}$ and $H_{\mathrm{z}}$ take minimum values.

At around $y=0$, note that the internal field exhibits a dramatic decrease from maximum to minimum. This means that the potential well is not only deep but also narrow. As a result, if an external perturbation at a relatively low frequency is imposed, the spins inside the well will be able to precess; however, those outside the well will not. In this way, the SDW causing the potential well should offer a narrow channel along which the SWs at subthreshold frequencies propagate and form a wave beam. This idea has been verified by micromagnetic simulations, and the results are presented in Figure 2.
Internal guiding of SWs due to the $1 \mathrm{D}$ confining potential Figure $2 \mathrm{a}$ shows the snapshots of SW distribution at certain frequencies in the magnonic waveguide with a SDW. From these figures, the formation of the SW beam is clear and justifies that the SDW in the waveguide can indeed serve as a channel to guide SW propagation. The well in the internal field is so deep that the SWs down to $2 \mathrm{GHz}$ can be present inside with much pronounced strength. In fact, the dispersion relation of the well mode suggests that the critical frequency $\left(f_{1}\right)$, above which SWs are permitted to exist in the channel, is close to zero (see Figure $2 \mathrm{~d}$ for details). When the excitation frequency exceeds $60 \mathrm{GHz}$, SWs will not only be trapped in the well but will also appear outside. That is, the barrier and well modes will be excited simultaneously (Figure 2a6). The SW wavelength of the well mode is much shorter than that of the barrier mode as a consequence of the difference in the internal field. As anticipated, the wavelength of the well-mode SW decreases as the frequency increases. In the well region, the magnetization is oriented along the $y$ axis and is thus perpendicular to the propagation direction of the SW, which forms the DE propagation geometry. It is well known that the DE SWs have positive dispersion and, more importantly, high group velocity in the allowed frequency range. ${ }^{42,43}$ Another striking feature in Figure $2 \mathrm{a}$ is that the leftward- and rightward-propagating SWs have different wavelengths and amplitudes, which indicates that the well-mode SWs along the internal channel are nonreciprocal. The nonreciprocity of SWs is an intrinsic property of the chiral magnetic system with an interfacial DMI. ${ }^{14,44}$ 


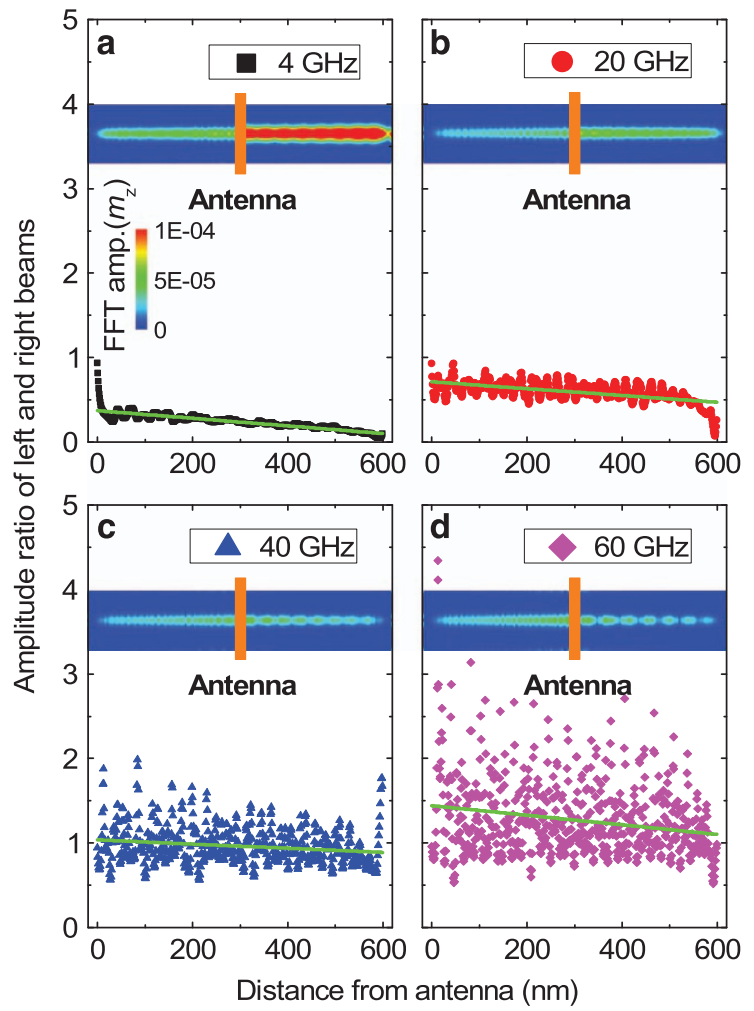

Figure 3 Channeled-SW attenuation with increased propagation distance. The lines are guides to the eyes and used to reflect the tendency for evolution of the amplitude ratio against the propagating distance. Inserted in each panel is the fast-Fourier-transform (FFT) amplitude distribution pattern, derived using SEMARGL software, ${ }^{29}$ of the channeled spin wave at the given frequency. (a) $4 \mathrm{GHz}$, (b) $20 \mathrm{GHz}$, (c) $40 \mathrm{GHz}$ and (d) $60 \mathrm{GHz}$.

As shown in Figure 2c, the beamwidth of the well-mode SW is almost independent of the frequency and is smaller than $10 \mathrm{~nm}$, which is in good agreement with what is expected from a well width at $\sim 10 \mathrm{~nm}$. Owing to the perfect confinement effect of the potential well, the strength of magnetization precession at the lateral edges is around zero. Accordingly, the SWs traveling along the channel are insensitive to the edge environment. In this way, we realize a self-cladding opticfiber-like magnonic waveguide. Here, the strongly modulated internal field is equivalent to the graded refractive index found in fiber optics, and the confining well plays a role like the core layer of an optic fiber. ${ }^{45}$ Potential devices built from such optic-fiber-like waveguides should possess high reliability and performance, as the negligible boundary scattering experienced by the channeled SWs will lead to an increased SW attenuation length and even coherence length.

For comparison, we examine SW propagation in the same waveguide but with a single-domain spin configuration (the left panel in Figure $2 b$ ). The right panels in Figure $2 b$ show the distribution of SWs at two characteristic frequencies. Below $58 \mathrm{GHz}$ or the threshold frequency $\left(f_{2}\right)$, no SWs can be excited in the waveguide. At $60 \mathrm{GHz}$ (slightly larger than $f_{2}$ ), only one edge mode can couple to the excitation field and propagate along the edge channels. At $80 \mathrm{GHz}$ (much larger than $f_{2}$ ), both of the two edge modes are activated. Obviously, these SWs are nonreciprocal, which is qualitatively consistent to what was found in the study by Garcia-Sanchez et al. ${ }^{14}$

The dispersion curves in Figure 2d can offer a full picture of the SW excitation and propagation characteristics in the waveguide. The bottom curve comes from the well mode in the SDW state, and the top curve comes from the edge-mode in the single-domain state. It is observed that any perturbation, with the upper frequency in the range of $f_{1}-f_{2}$, will be able to pass through the waveguide in the SDW state but will be stopped in the waveguide in the single-domain state. Namely, by toggling the magnetization state of the waveguide, the SWs can be switched on and off. This feature is explored to implement logic operations, as will be shown later. In contrast to the edge mode, the well mode exhibits an enhanced nonreciprocity (compare the dispersion curves in the range of $60-80 \mathrm{GHz}$ ). For the same frequency, the well mode has a lower phase velocity but a higher group velocity than the edge mode, which promises a larger SW coherence length.

The internal-field profiles for the SDW and single-domain spin textures are shown in the left and right panels of Figure 2e, respectively. One can see that the side wells stemming from the partial walls ${ }^{14}$ situated around the lateral edges (see the left panel of Figure $2 \mathrm{~b}$ ) are rather shallow compared with the center well created by the SDW. The field values corresponding to the side- and centerwell bottoms are approximately 16 and $2.5 \mathrm{kOe}$, respectively. This can account for the large difference in the open frequencies $\left(f_{2}-f_{1}\right)$ of the well and edge modes when considering that the open frequency of the mode excitation is determined by the strength of the effective field in the excitation region.

In Figure 2a, we have found that the channeled SWs are reciprocal, as revealed by the different wavelengths and amplitudes of the leftward and rightward traveling SWs. Because of the limited size of the waveguide, it is impossible to directly derive the attenuation lengths of these two beams; however, it is still possible to assess which of the two branches decays faster. Our idea is as follows: Discarding the phase information, the amplitude of propagating SWs as a function of the propagation distance is given by $A(x)=A_{0} \cdot \exp (-x / \Lambda),{ }^{44}$ where $A_{0}$ and $\Lambda$ are the excitation amplitude and attenuation length of a SW, respectively. Thus, the amplitude ratio of the leftward and rightward propagating SWs is $\eta=A^{l}(x) / A^{r}(x)=A_{0}^{l} / A^{r}{ }_{0} \cdot \exp \left[x \cdot\left(\Lambda_{\mathrm{l}}-\Lambda_{\mathrm{r}}\right) /\left(\Lambda_{\mathrm{l}} \cdot \Lambda_{\mathrm{r}}\right)\right]$. If $\Lambda_{l}=\Lambda_{r}$, then $\eta=A_{0}^{l} / A^{r}{ }_{0}=C$, which means that the amplitude ratio is constant and independent of the propagation distance. If $\Lambda_{\mathrm{l}}<\Lambda_{\mathrm{r}}$, then $\eta=C \cdot \exp (a \cdot x)$ where $a=\left(\Lambda_{\mathrm{l}}-\Lambda_{\mathrm{r}}\right) /\left(\Lambda_{\mathrm{l}} \cdot \Lambda_{\mathrm{r}}\right)<0$, suggesting that the amplitude ratio will decrease monotonically with the propagation distance; otherwise, it will increase monotonically with the propagation distance.

The amplitude ratio between the left and right beams against the propagation distance as well as the associated amplitude patterns for SWs at several characteristic frequencies are shown in Figure 3. From these figures, it is clear that the amplitude ratio is a decreasing function of the propagation distance. We thereby infer that $\Lambda_{1}<\Lambda_{\mathrm{r}}$, namely, the rightward traveling SWs decay at a slower rate than the leftward traveling ones. In addition, it is found that the amplitude ratio increases with the frequency, which implies that the higher the excitation frequency, the higher the relative excitation efficiency between the left and right beams. This behavior is already noticeable in Figure 2a.

Now that the SW channel is defined by the SDW that introduces the confining potential, is it possible to have multiple channels in a single waveguide containing more than one SDW? If so, those ultrathin magnetic samples with repeated strip domains at equilibrium, realized experimentally by different groups, ${ }^{9,24,25}$ might be used as a multichannel magnonic waveguide. To test this concept, we write two and three SDWs into one waveguide and then examine SW propagation in the waveguide with either of the written spin textures. From Figures $4 \mathrm{a}$ and $\mathrm{b}$, it is apparent that the 2-SDW and 3-SDW spin textures can statically exist in a single waveguide, and the excitation and propagation of SWs in the parallel confining wells are also 

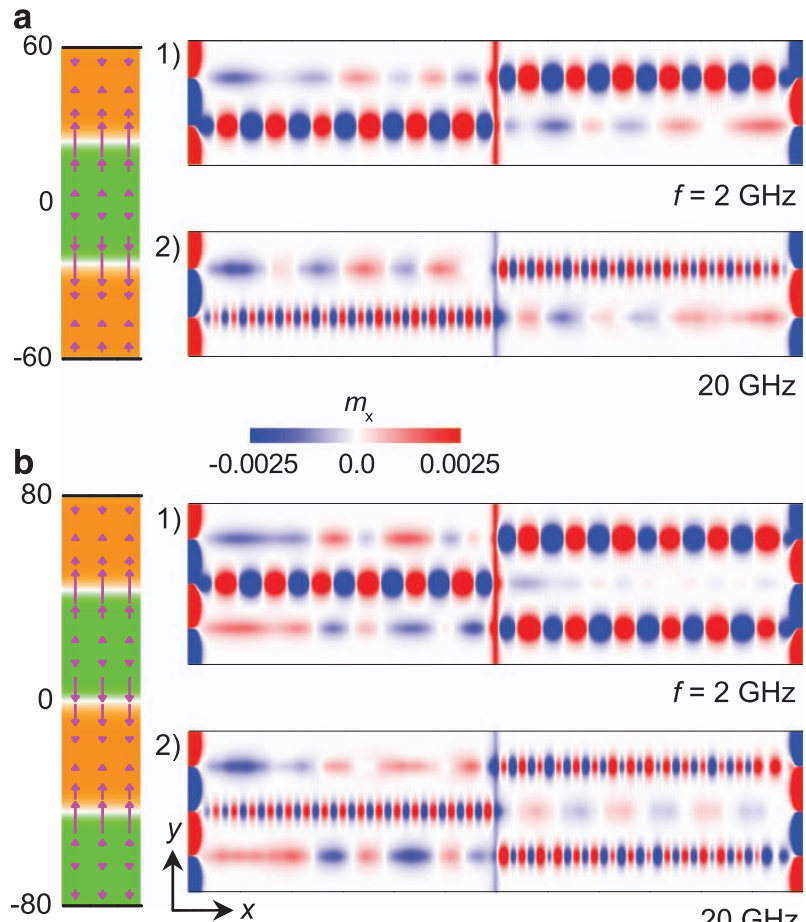

$20 \mathrm{GHz}$
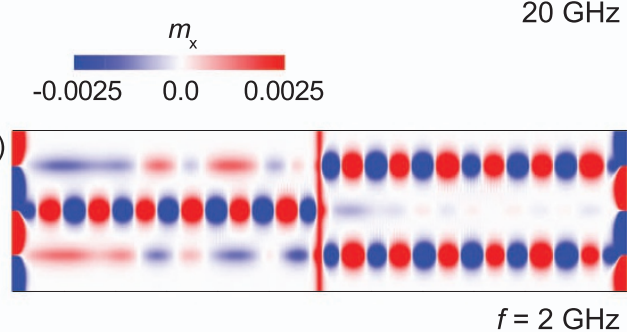

$-80$

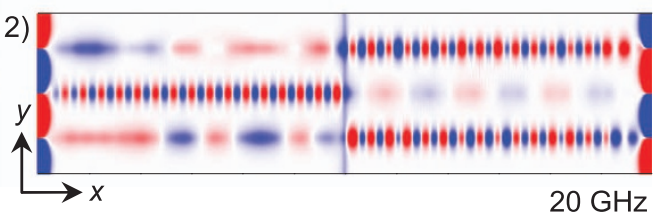

Figure 4 Realization of a multichannel waveguide based on a magnetic plate incorporating more than one SDW. Static spin configurations and steadystate SW propagation patterns for the (a) 2-SDW and (b) 3-SDW states are presented in the left and right panels, respectively. The magnetic plates are (a) 120 and (b) $160 \mathrm{~nm}$ in width. Both samples are $1200 \mathrm{~nm}$ long.

attainable dynamically. Because of the inherent nonreciprocity of the channeled SWs, the beams in neighboring channels exhibit different wave characteristics, that is, the wavelength, strength, attenuation length and so on. As a consequence, patterning of an extended film into 1D magnonic waveguides to confine SWs might be avoidable, and manipulation of the SW propagation for functional operations can possibly be done directly within individual channels written into the film rather than in separate waveguides patterned from the film. The channel properties can be tailored, for example, by engineering material or interface parameters. ${ }^{25,27}$ The spacing between nearby channels should be controllable by means of a local Oersted field, ${ }^{11}$ spin-transfer torque ${ }^{33}$ or spin-Hall torque ${ }^{46}$ (for details on how to control the spacing between nearby channels, see Supplementary Figure 5).

Logical operations based on state-modulated SW transmission To relate these findings to practical applications, we constructed magnonic logic gates on the basis of the state-controlled switch of the SWs in the novel waveguide. The structure of the logic-NOT gate (Figure $5 \mathrm{a}_{1}$ ) incorporates a short waveguide $(300 \mathrm{~nm}$ long, $60 \mathrm{~nm}$ wide) and a pair of antennas. The left antenna is used to inject SW signals into the waveguide, and the right antenna is used to read out these signal via inductive coupling. The logic input and output of this NOT gate are encoded into the spin state of the waveguide and the amplitude of the received signal, respectively. In principle, a logicNOT gate in combination with at least one additional two-input logic gate will enable the implementation of arbitrary logical operations, sometimes with the assistance of the fan-out and cross-over routing units. ${ }^{47}$ Here, we build a two-input logical NAND gate by connecting a

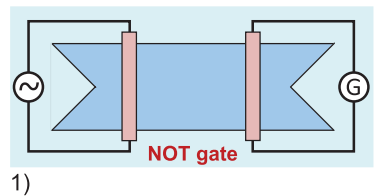

2)

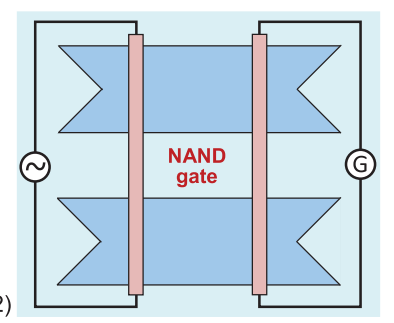

b
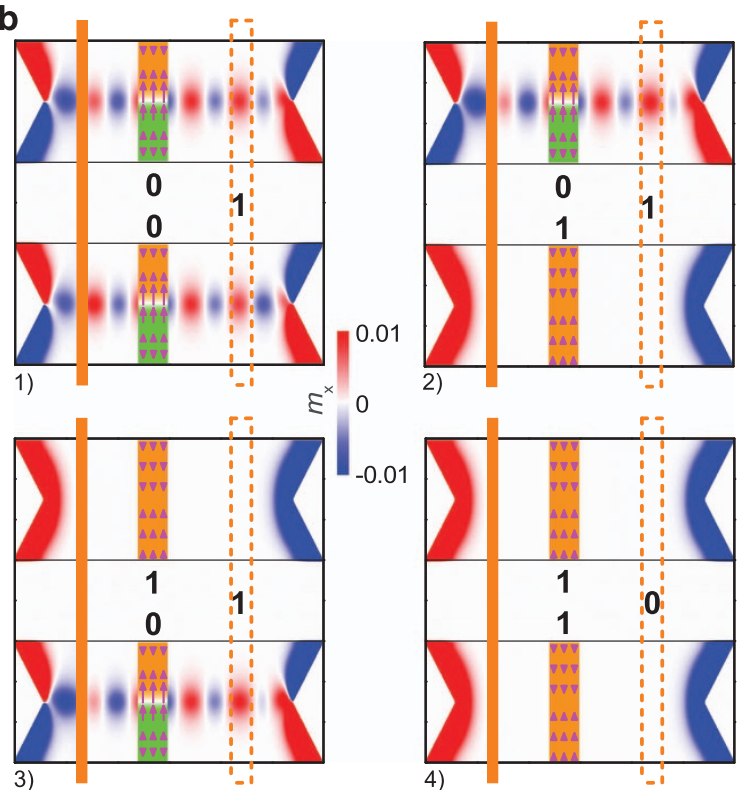

Figure 5 Logical NOT and NAND gates based on state-modulated SW transmission. (a) Diagram of the gate architecture. The left antenna is the SW source and the right antenna detects the output signal encoded into the SW strength. Logic input is encoded into the spin state of the gate's arm. The notches at the ends of a gate arm are used to reduce SW reflection. (b) Logic-NAND operation and truth table. In each arm, the background spin state and corresponding SW distribution are shown together. Each arm is $300 \mathrm{~nm}$ long and $60 \mathrm{~nm}$ wide; the separation between the two arms is $40 \mathrm{~nm}$. The frequency of the used spin wave is $10 \mathrm{GHz}$. Other separation values of 20, 60 and $80 \mathrm{~nm}$ were also examined in simulations to check if the dipolar coupling ${ }^{57}$ between the two arms will affect the operation of the NAND gate, and it was found that, for the chosen smallest separation $20 \mathrm{~nm}$, the NAND gate can still work well.

two such logic-NOT gates in parallel in a manner as depicted in Figure $5 \mathrm{a}_{2}$. The spin configurations in the two arms of the NAND gate can be manipulated independently in the operation process.

Figure $5 \mathrm{~b}$ shows the simulation results for the logic-NAND function. When the two arms are both in the SDW state (logic ' 0 ' input), the probe antenna will be penetrated by the strongest magnetic flux and give the largest inductive voltage (logic ' 1 ' output). When one of the two arms is converted to the single-domain state (logic ' 1 ' input), the magnetic flux entering into the receiver will be still nonzero, albeit decreased to half of the strongest strength (logic ' 1 ' output). Nevertheless, once both arms are set to the single-domain state, the detection antenna will collect nothing except noise (logic ' 0 ' output). This is the full detail of the logical NAND operations where the functionality of the NOT gate has also been proven.

The readout of data based on the inductive method is fast, and thus the operation speed relies mainly on the writing efficiency. The writing of the SDW can be achieved using conventional spin-transfer torque, ${ }^{33}$ emergent spin-Hall torque, ${ }^{46}$ or even fringing field as in the 
a1

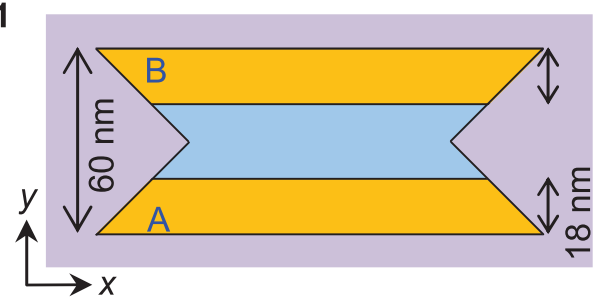

a2

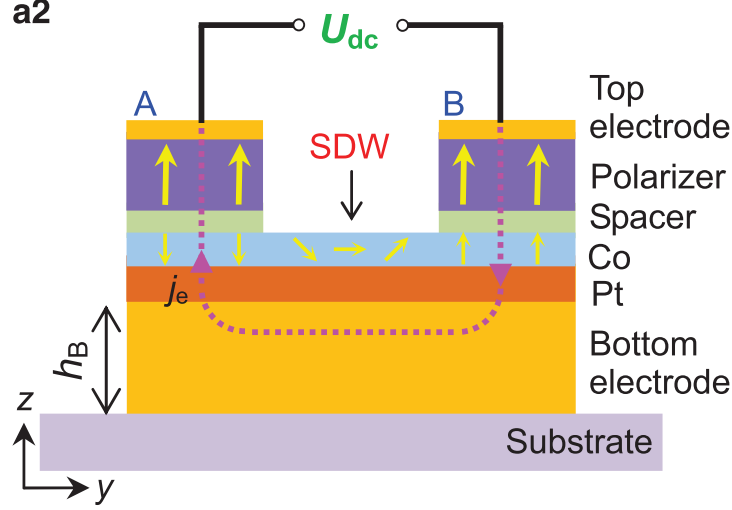

b
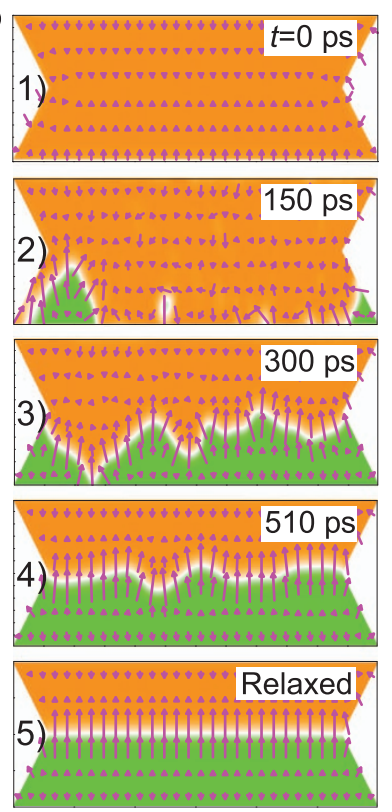

Figure 6 Writing a SDW into a gate arm. $\left(a_{1}\right)$ Top view and $\left(a_{2}\right)$ side view of the gate arm integrated with a writing unit. The writing unit and the Pt/Co waveguide layer form a trilayer spin-valve structure. The two top electrodes supplied with a voltage, $U_{\mathrm{DC}}$, cause an electron flow along the dotted line (the distribution of the current flowing lines in the $y z$ plane is illustrated in Supplementary Figure 6). Initially, both the polarizer and free layer are magnetically oriented upward. Under the action of opposite torques from the spin-polarized current, the spins in the Pt/Co layer underneath the A top electrode are reversed, whereas those below the B electrode maintain the initial orientation, which introduces a SDW in between. (b) Transient states at indicated times after current launching. We have checked the influence of the density of electron current $\left(J_{Z}\right)$ on the injection of the reverse domain by changing $J_{Z}$ from 0.9 to $2.0 \mathrm{MA} \mathrm{mm}^{-2}$ with an interval of $0.1 \mathrm{MA} \mathrm{mm}^{-2}$ and found that the reverse domain can be reliably written into the Pt/Co layer for $J_{z}$ not less than 1.1 $\mathrm{MA} \mathrm{mm}^{-2}$. In simulations, we focused only on spin dynamics of the free layer, and properties of the spacer and polarizer were modeled by several parameters. The spin polarization was assumed to be $0.4^{27}$ and the spin orientation in the polarizer is $m_{p}=(0,0,1)$, that is, along the $+Z$ axis. The effect of the out-of-plane (field-like) torque is negligible, as there is no difference in spin dynamics of the free layer for $\xi=0$ and 1.0 , where $\xi$ is the amplitude ratio of the field-like torque to the in-plane one. ${ }^{27}$

present-day hard-disk storage. Here, we give an example of injecting the SDW using a spin-polarized current, whose flowing path is defined by specially designed electrodes (see Figures $6 \mathrm{al}$ and a2). A pair of top electrodes supplied with a DC voltage $\left(U_{\mathrm{DC}}\right)$ will guide the electrons to flow along the dotted line. To polarize the current, a nonmagnetic spacer and a polarizer are attached atop the $\mathrm{Pt} / \mathrm{Co}$-free layer to form an inverted trilayer spin valve (see Figure 6a for details). Initially, both the polarizing and free layers have an upward magnetization. When the applied voltage is turned on, the spins in the $\mathrm{Pt} / \mathrm{Co}$ layer underneath the $\mathrm{A}$ and $\mathrm{B}$ top electrodes are subjected to opposite torques. Consequently, these spins below the A electrode will be reversed, whereas those below the B electrode will maintain the initial orientation. After relaxation, a SDW will be created in the central region of the $\mathrm{Pt} / \mathrm{Co}$ layer.

The whole dynamic process is shown in Figure 6b. At $t=0$, the current is switched on. Immediately, the reversed domain is nucleated at the lower boundary of the free layer as shown in Figure 6b2. The reversed domain expands with increasing current. At $\sim 510 \mathrm{ps,} \mathrm{a} \mathrm{zigzag}$ SDW is injected into the free layer. By relaxing the transient state under zero current, a regular SDW is formed in the interior of the free layer. Note that current injection with such dual top electrodes is key to reliable writing of a SDW into the Pt/Co waveguide (that is, the free layer). Using only the A, top electrode will result in plenty of randomness in the injection of the SDW. In the presence of a $\mathrm{B}$ electrode, the spins below it will be well stabilized by the passing current during the spin switch underneath the A electrode. On the other hand, the usage of such a dual-electrode structure will introduce an undesirable in-plane current in the Co layer as denoted by the magenta dotted line in Figure $6 \mathrm{a} 2$ and Supplementary Figure 6. However, the bottom electrode could experimentally be made far thicker than the Pt/Co layer in real devices so that the density of the in-plane current $\left(J_{\mathrm{y}}\right)$ flowing through the Pt and Co layers and the bottom-electrode layer is much lower than that of the perpendicular current $\left(J_{\mathrm{Z}}\right)$; this would result in negligible spin-transfer (Zhang-Li form $^{48}$ ) and spin-Hall torques ${ }^{46}$ on magnetic moments in the Co layer. Consequently, the nucleated domain wall structure should not be remarkably affected by the transverse current (see Supplementary Figure 7 for the full detail). The notches at the ends of the waveguide have two effects: they can favor nucleation of the reversed domain and suppress the reflection of SWs at the boundary.

The energy consumed to write the SDW (a bit) into the waveguide (a logic-NOT arm) is $\sim 0.1 \mathrm{pJ}$ (the detail for estimating the write energy is presented in section III of the Supplementary Information), which is comparable to values recently reported for spin-transfer torque magnetic random access memory (STT-MRAM) constructed with spin-valve nanopillars ${ }^{49}$ or magnetic tunnel junctions, ${ }^{50}$ novel magnetic random access memory (MRAM) based on planar magnetostrictive nanostructures, ${ }^{51}$ ferroelectric random access memory $(\text { FeRAM })^{52}$ and so on.

\section{DISCUSSION}

Finally, we would like to make additional remarks on the advantages of the proposed waveguide. The channeling effect of the waveguide relies on the potential well arising from the existence of a SDW. The 
internal field is inhomogeneous and gives a 1D deep potential well across the waveguide; however, it is continuous and uniform in the longitudinal direction of the waveguide. Accordingly, the SWs in the waveguide are confined transversely and flow along the SDW-defined channel, which form an ultra-narrow (10 nm, deep submicrometer) beam with the beamwidth determined by the well width.

Here, the SDW-induced confinement effect is different in nature from the boundary-induced one in patterned long waveguides. ${ }^{23,53}$ For patterned $1 \mathrm{D}$ waveguides in the $\mathrm{DE}$ propagation geometry, the inhomogeneous internal field produces two potential wells centered at the lateral physical edges and one potential barrier at the core of a waveguide. This causes an increasing refractive index from the core of the waveguide, ${ }^{54}$ which might lead to extra dispersion ${ }^{55}$ of the SWs because of multimode coexistence and beating. ${ }^{43}$ Moreover, the unwanted scattering of the center mode by unavoidable edge roughness, owing to the nonzero precession amplitude at the edges, will also cause undesirable decay. ${ }^{13}$

With regard to our proposed waveguide, the potential well lies in the interior of the waveguide, wherein the effective field assumes the lowest value over the waveguide width and the refractive index is thereby the largest. In the frequency window of $f_{1}-f_{2}(\sim$ several tens of $\mathrm{GHz}$ ), no mode excitation is allowed outside of the middle channel. That is, no multimode beating and edge scattering can occur in the boundary areas. This feature is expected to suppress mode dispersion and enlarge the attenuation length of the signal wave; thus, it is highly desired in wave-based real devices.

Apart from the demonstrated application in channeling SWs, more potential applications of the SDWs or SDW-induced wells in other aspects can also be enumerated. For instance, the SDW can behave as a virtual magnetic boundary, beside which the internal field is highly inhomogeneous and SW Goos-Hanchen effect ${ }^{55}$ is anticipated to occur and benefit from the roughness-free character of this boundary. In domain regions away from the 'SDW boundary', the magnetization is normal to the sample surface, and thus the forward-volume SW propagation geometry ${ }^{42}$ is fulfilled. It is easier to derive the GoosHanchen shift at the boundary for the isotropic SW dispersion in this propagation geometry than for the anisotropic SW dispersion in the other propagation geometries. In addition, magnetic samples, in which an array of SDWs are packed periodically and in parallel, should play the role of a magnonic bandgap crystal ${ }^{56}$ because of the periodicity of the internal field in the sample. The sharp contrast between the SDW and strip-domain regions in the effective field promises a large magnonic band gap. ${ }^{1}$

In conclusion, we demonstrate by means of micromagnetic simulations that chiral SDWs in ultrathin magnets with induced DMI can cause a deep potential well $10 \mathrm{~nm}$ in width. Because of the continuity and uniformity of the well along the SDW, guided propagation of SWs along the SDW can be achieved. As a result, an ultra-narrow $(\sim 10 \mathrm{~nm})$ SW channel is created at the interior of a waveguide composed of chiral magnets. In this way, we realize a selfcladding optic-fiber-like magnonic waveguide with 'graded refractive index'. This kind of waveguide is advantageous in several aspects compared with the frequently used width-confined SW waveguides. ${ }^{53}$ First, it can avoid the boundary scattering ${ }^{13}$ caused by edge irregularity and the extra dispersion ${ }^{55}$ because of multimode confluence and beating in the width-confined waveguides. ${ }^{53}$ In addition, the SDW state leading to the confining well and thus the channeling effect is nonvolatile, that is, its presence does not rely on an applied field. Based on the state-modulated SW propagation in the proposed waveguide, we build logical NOT and NAND gates whose input and output are coded into the spin state of the gate's arm and the amplitude of the signal SW, respectively. We also developed an advanced writing scheme capable of reliably writing a SDW via delicately distributed current-injection pads. The present study opens up a route toward interconnection of the magnonic logic circuits and skyrmion-based racetrack memories. ${ }^{27,28}$

\section{CONFLICT OF INTEREST}

The authors declare no conflict of interest.

\section{ACKNOWLEDGEMENTS}

XJX is financially supported by the Zhejiang Provincial Natural Science Foundation of China under Grant No. LY14A040006 and by the National Natural Science Foundation of China under the Grant No. 11104206. YZ acknowledges the support by the National Basic Research Program of China (Grant No. 2014CB921101), the NSFC (Grants No. 61274102 and No. 61427812) of China, the support by the Seed Funding Program for Basic Research and Seed Funding Program for Applied Research from the HKU, ITF Tier 3 funding (ITS/171/13), the RGC-GRF under Grant HKU 17210014 and University Grants Committee of Hong Kong (Contract No. AoE/P-04/08).

Author contributions: Both authors conceived the study. XJX performed the micromagnetic simulations. Both authors analyzed the micromagnetics simulation results and prepared the manuscript.

1 Lenk, B., Ulrichs, H., Garbs, F. \& Munzenberg, M. The building blocks of magnonics. Phys. Rep. 507, 107-136 (2011).

2 Khitun, A., Bao, M. \& Wang, K. L. Magnonic logic circuits. J. Phys. D Appl. Phys. 43, 264005 (2010).

3 Dzyaloshinskii, I. E. Thermodynamic theory of 'weak' ferromagnetism in antiferromagnetic substances. Sov. Phys. JETP 5, 1259-1272 (1957).

4 Moriya, T. Anisotropic superexchange interaction and weak ferromagnetism. Phys. Rev. 120, 91-98 (1960).

5 Fert, A. Magnetic and transport properties of metallic multilayers. Mater. Sci. Forum 59-60, 439-480 (1990).

6 Kruglyak, V. V., Gorobets, O. Y., Gorobets, Y. I. \& Kuchko, A. N. Magnetization boundary conditions at a ferromagnetic interface of finite thickness. J. Phys. Condens. Matter 26 406001 (2014).

7 Bode, M., Heide, M., von Bergmann, K., Ferriani, P., Heinze, S., Bihlmayer, G., Kubetzka, A., Pietzsch, O., Blügel, S. \& Wiesendanger, R. Chiral magnetic order at surfaces driven by inversion asymmetry. Nature 447, 190-193 (2007).

8 Heide, M., Bihlmayer, G. \& Blügel, S. Dzyaloshinskii-Moriya interaction accounting for the orientation of magnetic domains in ultrathin films: Fe/W(110). Phys. Rev. B 78, 140403 (2008)

9 Chen, G., Zhu, J., Quesada, A., Li, J., N'Diaye, A. T., Huo, Y., Ma, T. P., Chen, Y., Kwon, H.Y., Won, C., Qu, Z.Q., Schmid, A.K. \& Wu, Y.Z. Novel chiral magnetic domain wall structure in Fe/Ni/Cu(001) films. Phys. Rev. Lett. 110, 177204 (2013).

10 Nagaosa, N. \& Tokura, Y. Topological properties and dynamics of magnetic skyrmions. Nat. Nano 8, 899-911 (2013).

11 Vogt, K., Fradin, F., Pearson, J., Sebastian, T., Bader, S., Hillebrands, B., Hoffmann, A \& Schultheiss, H. Realization of a spin-wave multiplexer. Nat. Commun 5, 3727 (2014).

12 Hertel, R., Wulfekel, W. \& Kirschner, J. Domain-wall induced phase shifts in spin waves. Phys. Rev. Lett. 93, 257202 (2004).

13 Duerr, G., Thurner, K., Topp, J., Huber, R. \& Grundler, D. Enhanced transmission through squeezed modes in a self-cladding magnonic waveguide. Phys. Rev. Lett. 108, 227202 (2012).

14 Garcia-Sanchez, F., Borys, P., Vansteenkiste, A., Kim, J. V. \& Stamps, R. L. Nonreciprocal spin-wave channeling along textures driven by the DzyaloshinskiiMoriya interaction. Phys. Rev. B 89, 224408 (2014).

15 Davies, C. S., Francis, A., Sadovnikov, A. V., Chertopalov, S. V., Bryan, M. T., Grishin, S. V., Allwood, D. A., Sharaevskii, Y. P., Nikitov, S. A. \& Kruglyak, V. V. Towards graded-index magnonics: Steering spin waves in magnonic networks. Phys. Rev. B 92, 020408(R) (2015).

16 Neusser, S. \& Grundler, D. Magnonics: Spin waves on the nanoscale. Adv. Mater. 21, 2927-2932 (2009).

17 Kruglyak, V. V., Demokritov, S. O. \& Grundler, D. Magnonics. J. Phys. D: Appl. Phys 43 264001 (2010).

18 Duerr, G., Huber, R. \& Grundler, D. Enhanced functionality in magnonics by domain walls and inhomogeneous spin configurations. J. Phys. Condens. Matter 24 024218 (2012).

19 Zakeri, K., Zhang, Y., Prokop, J., Chuang, T. H., Sakr, N., Tang, W. X. \& Kirschner, J. Asymmetric spin-wave dispersion on Fe(110): Direct evidence of the DzyaloshinskiiMoriya interaction. Phys. Rev. Lett. 104, 137203 (2010). 
20 Cho, J., Kim, N.-H., Lee, S., Kim, J.-S., Lavrijsen, R., Solignac, A., Yin,, Y., Han,, D. S., van Hoof,, N. J., Swagten,, H. J., Koopmans,, B. \& You,, C.Y. Thickness dependence of the interfacial Dzyaloshinskii-Moriya interaction in inversion symmetry broken systems. Nat. Commun. 6, 7635 (2015).

21 Damon, R. W. \& Eshbach, J. R. Magnetostatic modes of a ferromagnet slab. J. Phys. Chem. Solids 19, 308-320 (1961).

22 Demidov, V. E., Demokritov, S. O., Rott, K., Krzysteczko, P. \& Reiss, G. Nanooptics with spin waves at microwave frequencies. Appl. Phys. Lett. 92, 232503 (2008).

23 Guslienko, K. Y., Demokritov, S. O., Hillebrands, B. \& Slavin, A. N. Effective dipolar boundary conditions for dynamic magnetization in thin magnetic stripes. Phys. Rev. B 66, 132402 (2002).

24 Kubetzka, A., Bode, M., Pietzsch, O. \& Wiesendanger, R. Spin-polarized scanning tunneling microscopy with antiferromagnetic probe tips. Phys. Rev. Lett. 88, 057201 (2002).

25 Chen, G., Ma, T., N'Diaye, A. T., Kwon, H., Won, C., Wu, Y. \& Schmid,, A. K. Tailoring the chirality of magnetic domain walls by interface engineering. Nat. Commun. $\mathbf{4}$, 2671 (2013)

26 Parkin, S. S. P., Hayashi, M. \& Thomas, L. Magnetic domain-wall racetrack memory. Science 320, 190-194 (2008).

27 Sampaio, J., Cros, V., Rohart, S., Thiaville, A. \& Fert, A. Nucleation, stability and current-induced motion of isolated magnetic skyrmions in nanostructures. Nat. Nano 8 , 839-844 (2013).

28 Zhou, Y. \& Ezawa, M. A reversible conversion between a skyrmion and a domain-wall pair in a junction geometry. Nat. Commun 5, 4652 (2014).

29 Dvornik, M., Au, Y. \& Kruglyak, V. V. Micromagnetic simulations in magnonics. Top. Appl. Phys. 125, 101-115 (2013).

30 Vansteenkiste, A., Leliaert, J., Dvornik, M., Helsen, M., Garcia-Sanchez, F. \& Van Waeyenberge, B. The design and verification of MuMax3. AIP Adv. 4, 107133 (2014).

31 Donahue, M. J. \& Porter, D. G. OOMMF User's Guide Version 1.0 Interagency Report NISTIR 6376, National Institute of Standards and Technology: Gaitherburg, MD, (1999).

32 Rohart, S. \& Thiaville, A. Skyrmion confinement in ultrathin film nanostructures in the presence of Dzyaloshinskii-Moriya interaction. Phys. Rev. B 88, 184422 (2013).

33 Slonczewski, J. Current-driven excitation of magnetic multilayers. J. Magn. Magn. Mater. 159, L1-L7 (1996).

34 Brown, W. F. Thermal fluctuations of a single-domain particle. J. Appl. Phys. 34, 1319-1320 (1963)

35 Shaw, J. M., Nembach, H. T. \& Silva, T. J. Resolving the controversy of a possible relationship between perpendicular magnetic anisotropy and the magnetic damping parameter. Appl. Phys. Lett. 105, 062406 (2014).

36 Tomasello, R., Martinez, E., Zivieri, R., Torres, L., Carpentieri, M. \& Finocchio, G. A strategy for the design of skyrmion racetrack memories. Sci. Rep 4, 6784 (2014).

37 Berkov, D. V. \& Gorn, N. L. Micromagnetic simulations of the magnetization precession induced by a spin-polarized current in a point-contact geometry. J. Appl. Phys. 99, 08Q701 (2006)

38 Iwasaki, J., Mochizuki, M. \& Nagaosa, N. Current-induced skyrmion dynamics in constricted geometries. Nat. Nano 8, 742-747 (2013).

39 Fujita, N., Inaba, N., Kirino, F., Igarashi, S., Koike, K. \& Kato, H. Damping constant of Co/Pt multilayer thin-film media. J. Magn. Magn. Mater. 320, 3019-3022 (2008).

40 Iwasaki, J., Beekman, A. J. \& Nagaosa, N. Theory of magnon-skyrmion scattering in chiral magnets. Phys. Rev. B 89, 064412 (2014).

41 Braun, H.-B. Fluctuations and instabilities of ferromagnetic domain-wall pairs in an external magnetic field. Phys. Rev. B. 50, 16485-16500 (1994).
42 Stancil, D. D. \& Prabhakar, A. Spin Waves Theory and Applications, Springer, (2009). 43 Kostylev, M., Gubbiotti, G., Hu, J. G., Carlotti, G., Ono, T. \& Stamps, R. Dipoleexchange propagating spin-wave modes in metallic ferromagnetic stripes. Phys. Rev. B 76, 054422 (2007).

44 Moon, J.-H., Seo, S.-M., Lee, K.-J., Kim, K.-W., Ryu, J., Lee, H.-W., McMichael, R. D. \& Stiles, M. D. Spin-wave propagation in the presence of interfacial Dzyaloshinskii-Moriya interaction. Phys. Rev. B 88, 184404 (2013).

$45 \mathrm{Kao}, \mathrm{C}$. K. Sand from centuries past: Send future voices fast Nobel Lecture http://www.nobelprize.org/nobel_prizes/physics/laureates/2009/kao_lecture.pdf (2009).

46 Liu, L., Lee, O. J., Gudmundsen, T. J., Ralph, D. C. \& Buhrman, R. A. Current-induced switching of perpendicularly magnetized magnetic layers using spin torque from the spin Hall effect. Phys. Rev. Lett. 109, 096602 (2012).

47 Allwood, D. A., Xiong, G., Faulkner, C. C., Atkinson, D., Petit, D. \& Cowburn, R. P. Magnetic domain-wall logic. Science 309, 1688-1692 (2005).

48 Zhang, S. \& Li, Z. Roles of nonequilibrium conduction electrons on the magnetization dynamics of ferromagnets. Phys. Rev. Lett. 93, 127204 (2004).

49 Bedau, D., Liu, H., Bouzaglou, J.-J., Kent, A. D., Sun, J. Z., Katine, J. A., Fullerton, E. E. \& Mangin, S. Ultrafast spin-transfer switching in spin valve nanopillars with perpendicular anisotropy. Appl. Phys. Lett. 96, 022514 (2010).

50 Zhao, H., Lyle, A., Zhang, Y., Amiri, P. K., Rowlands, G., Zeng, Z., Katine, J., Jiang, H., Galatsis, K., Wang, K. L., Krivorotov, I. N. \& Wang, J.-P. Low writing energy and sub nanosecond spin torque transfer switching of in-plane magnetic tunnel junction for spin torque transfer random access memory. J. Appl. Phys. 109, 07 C720 (2011).

51 Ostler, T. A., Cuadrado, R., Chantrell, R. W., Rushforth, A. W. \& Cavill, S. A. Strain induced vortex core switching in planar magnetostrictive nanostructures. Phys. Rev. Lett. 115, 067202 (2015).

52 International Technology Roadmap for Semiconductors, 2010 Future Memory Devices Workshop Summary http://www.itrs.net/.

53 Demokritov, S. O., Demidov, V. E. in Spin Wave Confinement (eds Demokritov S. O.) Chap. 1 (Pan Stanford Publishing Pte. Ltd., Singapore, 2009).

54 Jorzick, J., Demokritov, S. O., Hillebrands, B., Bailleul, M., Fermon, C., Guslienko, K. Y., Slavin,, A.N., Berkov,, D.V. \& Gorn,, N.L. Spin wave wells in nonellipsoidal micrometer size magnetic elements. Phys. Rev. Lett. 88, 047204 (2002).

55 Gruszecki, P., Romero-Vivas, J., Dadoenkova, Y. S., Dadoenkova, N. N., Lyubchanskii, I. L. \& Krawczyk, M. Goos-Hänchen effect and bending of spin wave beams in thin magnetic films. Appl. Phys. Lett. 105, 242406 (2014).

56 Wang, X.-G., Guo, G.-H., Li, Z.-X., Wang, D.-W., Nie, Y.-Z. \& Tang, W. Spin-wave propagation in domain wall magnonic crystal. Europhys. Lett. 109, 37008 (2015).

57 Wang, Z. K., Lim, H. S., Zhang, V. L., Goh, J. L., Ng, S. C., Kuok, M. H., Su,, H.L. \& Tang, S.L. Collective spin waves in high-density two-dimensional arrays of FeCo nanowires. Nano Lett. 6, 1083-1086 (2006).

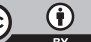

This work is licensed under a Creative Commons Attribution 4.0 International License. The images or other third party material in this article are included in the article's Creative Commons license, unless indicated otherwise in the credit line; if the material is not included under the Creative Commons license, users will need to obtain permission from the license holder to reproduce the material. To view a copy of this license, visit http:// creativecommons.org/licenses/by/4.0/

Supplementary Information accompanies the paper on the NPG Asia Materials website (http://www.nature.com/am) 